67

\section{HUMAN BAUPLAN ANOMALIES}

P. CINCINNATI ${ }^{1}$ AMBULATORIO DI GENETICA CLINICA, U.O.C. DI PEDIATRIA E NEONATOLOGIA, OSPEDALE E. DE SANTIS, GENZANO DI ROMA (ITALY)

The german term Bauplan means body-plan, but captures also the idea of the architectura constraints existing in such a functional design. There are only a few animal Bauplane, and each phylum is characterized by one of them. Major Bauplan's modifications within a taxon are no tolerated. The concept is common between the zoologists, but usually is not employed in human pathology. Our hypothesis is that an approach to the birth defects in terms of basic body-plan results in a better understanding of the human ontogenetic and phylogenetic development. We observed 30 interrupted pregnancies owing to severe structural fetal malformations. A pathological study was performed in all the fetuses. We found the following syndromes and anomalies: Meckel-Gruber syndrome 1; Cantrell's pentalogy 1; Ivemark syndrome 1; Omphalocele-radial hypoplasia complex 1 Triploid syndrome 1; Malformative associations including encephalic anomalies 3; SNC isolated abnormalities (comprehensive of 10 neural tube defects) 16; Gastroschisis 1; Complex cardiac anomalies 3; Kidney defects, including bilateral renal agenesis 2; According to our study, three major Bauplan defects are detectable in the aborted fetuses. They are concerning with: (1) cephalization, (2) laterality, (3) closure of the fetal body surface, and all of them are related to a midline pathology. These results are in agreement with the belonging of our species to the chordates deuterostomata and suggest that the human Bauplan begins to appear in the early blastogenesis, when the nothocord appears an neurulation occurs. In this period the embryo seems to act as a single morphogenetic unit, so that blastogenetic defects are frequently multisystem abnormalities. 1/3 of the fetuses presented complex malformative disorders. Besides being a source for studies of evolutionary developmental biology (Evo-Devo), the investigation on human blastogenesis and organogenesis through an oriented evaluation of the birth defects could have in the future unexpected therapeutic relevance.

\section{8}

MARKED STATEWIDE VARIATION IN RISK-ADJUSTED HOSPITAL CHARGES FOR CONGENITAL HEART SURGERY

J CONNOR $^{l}, K$ GAUVREAU ${ }^{l}, K$ JENKINS ${ }^{l}{ }^{\text {I}}$ CHILDREN'S HOSPITAL BOSTON (USA)

Background: Selected referral to institutions with low mortality rates has been proposed as a strategy to improve outcomes for congenital heart surgery; the impact of such strategies in medical costs is not known.

Objective: To examine whether total hospital charges for congenital heart surgery varies across geographic regions

Methods: Cases of congenital heart surgery $(<18 \mathrm{y})$ were identified from the Health Care Utilization Project KID 2000 database (27 states) using ICD-9-CM codes. To adjust for baseline case mix differences, patient characteristics independently associated with higher hospital charges were identified. Procedures were grouped into RACHS-1 risk categories. Reported total hospital charges were log transformed. Differences between observed and expected mean total hospital charges were examined transformed. Differences between observed and expected mean total hospital charges were examined
for each state; predictors of these differences were explored. Proportions of state volume of congenital heart surgery, proportions of caseload at children's and teaching hospitals, and complexity of case mix by insurance were also examined.

Results: Among the 9,406 cases of congenital heart surgery for which a RACHS-1 risk category could be assigned, mean total hospital charges were $\$ 54,725$. RACHS-1 risk category, age, prematurity, major non-cardiac structural anomaly, multiple surgical procedures, chromosomal abnormality and weekend admisiion were associated with higher charges. Among the 27 states examined, charges varied by $\$ 51,912(\$ 24,020$ to $-\$ 27,892)$. Institutional and case mix descriptors were not found to be associated with the average dollar charge differential.

Conclusions: States vary considerably in risk adjusted total hospital charges for congenital heart surgery procedures. These variations could not be explained by state volume of congenital heart surgery cases per number of institutions performing congenital heart surgery, proportions of caseload at children's and teaching hospitals, and complexity of case mix by insurance.

\section{9}

ECONOMIC BURDEN OF CONGENITAL HEART DISEASE ON FAMILIES

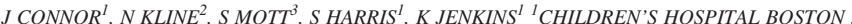
${ }^{2}$ MEMORIAL SLOAN KETTERING CANCER CENTER, ${ }^{3}$ BOSTON COLLEGE (USA)

Objective: The economic burden of pediatric illness and its social impact on families has not been described in the area of congenital heart disease (CHD). The purpose of this study was to describe the economic burden of CHD as experienced by families.

Methods: An exploratory inquiry using semi-structured interviews was conducted. A pruposive sampling technique was use to identify parents of children with varying CHD complexity and socioeconomic status (SES) admitted for congenital heart surgery at a tertiary care center. Complexity of CHD was approximated using the Risk Adjustment for Congenital Heart Surgery Method (RACHS of CHD was approximated using the Risk Adjustment for Congenital Heart Surgery Method (RACHS
1). SES was estimated using Hollingshead's Four Factor Index Scale. Structured interviews guided by 1). SES was estimated using Hollingshead's Four Factor Index Scale. Structured interviews guided by
6 questions focusing on the meaning of cost and the impact on social functioning on the family were conducted. Interviews lasted 45 minutes to 1 hour. All interviews were auditaped and transcribed verbatim. Data were numbered, sorted, and coded using NVIVO software. Three researchers independently examined the data to identify thematic categories.

Results: Of the 20 interviews, the meaning of cost resulted in the emergence of two themes, uncertainty and lifestyle change. Data could be further sorted into 3 underlying categories of financial, emotional and family burden. Inter-rater reliability was $91 \%$. As a result of increased disease complexity, families seem to experience a high level of stress in terms of finances, emotional drain, and family member burden. Families who received a prenatal diagnosis verbalized economic burden as an early stressor and altered personal spending prior to birth. Perception of the economic impact of the child's condition was dependent on baseline SES.

Conclusion: Although the complexity of disease seems to increase economic burden, timing of diagnosis and baseline SES were also noted to contribute to economic stress.
70

MEASURING RELATIVE QUALITY AND RESOURCE USE FOR CONGENITAL HEART SURGERY

J CONNOR ${ }^{1}, K$ GAUVREAU ${ }^{1}, K$ JENKINS ${ }^{1}{ }^{1}$ CHILDREN'S HOSPITAL BOSTON (USA)

Objective: Tto develop a composite mesure reflecting both quality and resource use for pediatric heart surgery and to examine regional variation for this measure.

Methods: Cases of congenital heart surgery $<18 \mathrm{y}$ were identified from the Health Care Utilization Project KID 2000 database (26 states) using ICD-9-CM codes. Cases meeting criteria for the Risk Adjustment for Congenital Heart Surgery method (RACHS-1) were placed into risk categories. Relative quality and resource use was examined using the product of the standardized mortality ratio (SMR) and standardized charge ratio (SCR), termed the Efficiency Product. For each state, SMR was defined as observed/expected mortality and SCR as observed/expected mean charges; expected values adjusted for baseline case mix differences using RACHS-1 risk category and additional patien characteristics. A state with mortality and charges both equal to expected would have an Efficiency Product equal to 1 . The relationship between mean charge differential (observed minus expected mean charges) and SMR for each state was also examined.

Results: Among 9,406 cases of congenital heart surgery, the mortality rate was $4.1 \%$ and the average median charges was $\$ 49,722$. Across the 26 states, the mean charge differential per state ranged from $-427,892$ to $+\$ 24,020$. Mortality rates per state ranged from $0.6 \%$ to $6.1 \%$. The SMR ranged from .13 to 1.87 and the SCR from .47 to 1.4 . The Efficiency Product ranged from .12 to 1.86 ( 6 states $<0.05$, 9- 0.05 to $.99,7-1.00$ to 1.49 , and $4->1.50$ ). There was no correlation between SMR and mean charge differential.

Conclusions: States varied considerably in a risk-adjusted measure of quality and resource use for congenital heart surgery procedures. This novel approach of assessing efficiency in this population may be the first step in identifying optimal charge structures for delivering care.

\section{1}

\section{DIFFUSION TRACTOGRAPHY OF
DEVELOPING PRETERM BRAIN}

SJ COUNSELL $L^{1}$ L SRINIVASAN ${ }^{1}$ IP BOARDMAN ${ }^{2}$ DJ LARKMAN ${ }^{l}$ IM ALLSOP ${ }^{1}$ IA FITZ PATRICK ${ }^{1}, F M$ COWAN ${ }^{2}, J V$ HAJNAL ${ }^{1}$, AD EDWARDS ${ }^{1,2}$, MA RUTHERFORD ${ }^{1,2}$ ROBERT STEINER MR UNIT, IMAGING SCIENCES DEPT, IMPERIAL COLLEGE LONDON, HAMMERSMITH HOSPITAL, ${ }^{2}$ DEPARTMENT OF PAEDIATRICS, IMPERIAL COLLEGE LONDON, HAM MERSMITH HOSPITAL (UK)

Background The emerging technique of diffusion tractography offers the opportunity to visualise white matter tracts in vivo. The aims of this study were to i. visualise and quantify developmental changes in the corticospinal tracts in the preterm brain using diffusion tractography ii. determine whether abnormalities in tract development associated with focal pathology can be identified in these infants.

Methods Subjects: High resolution DTI was obtained on 21 preterm infants who were born at a median (range) gestational age (GA) of $29(26-34)$ weeks and imaged at a median post-menstrual age (PMA) of 38 (30 - 44) weeks. 17 infants had no evidence of abnormality on MRI and 4 infants had focal lesions. Magnetic resonance imaging: MRI was performed on a 3 Tesla Philips MR system. DTI was acquired in 15 non-colinear directions with a b value of $750 \mathrm{~s} / \mathrm{mm} 2$ and an image voxel size of $1.75 \times 1.75 \times 2 \mathrm{~mm} 3$. Tractography: Diffusion tractography was performed using fibre assignment by continuous tracking (FACT). Linear regression analysis was performed to test the relationship between mean fibre length, fractional anisotropy (FA) and apparent diffusion coefficient (ADC) and PMA at scan in the infants who had no evidence of abnormality on MRI. In addition, tractography data in infants who had focal lesions were compared to 7 infants with no lesions at term equivalent age (PMA $\mathrm{p}=0.94)$

Results Mean extracted fibre length $(\mathrm{p}<0.001)$ and FA increased $(\mathrm{p}<0.001)$ and ADC decreased $(\mathrm{p}<0.001)$ with increasing PMA. Mean fibre length $(\mathrm{p}=0.01)$ and FA $(\mathrm{p}=0.005)$ were reduced in infants with focal lesions.

Conclusions Diffusion tractography can visualise and quantify the developing corticospinal tracts in the preterm brain. These initial results suggest that tractography may be a useful tool for identifying anomalies in tract development associated with pathology.

\section{2}

RELEVANCE OF SYMPTOMS VERSUS OBJECTIVE EVIDENCES IN NEONATAL GASTROESOPHAGEAL REFLUX DISEASE

F CRESI ${ }^{1}$, F SAVINO, C MARINACCIO, A TESTA, MC RUSSO, L DE SANCTIS, L SILVESTRO ${ }^{1}$ UNIVERSITY OF TURIN (ITALY)

Background: Gastroesophageal reflux disease (GERD) in newborn is characterized by a protean symptomatology that includes excessive irritability and crying, frequent vomiting and regurgitations, feeding problems, rumination, apnea, aspiration pneumonia, failure to thrive and anemia. The aim of this study was to investigate the relationship between symptoms and gastroesophageal activity in infants with gastroesophageal reflux.

Methods: To evaluate gastroesophageal tract activity we have combined multichannel intraluminal electrical impedance (MII) and $\mathrm{pH}$ monitoring with epigastric impedance (EGI) in 30 neonates with suspected GERD. This combination of three methods provides complementary, simultaneous data from the esophagus and stomach. MII detected reflux episodes were characterized by time of occurrence, duration, height, $\mathrm{pH}$, gastric filling state and gastric emptying velocity. Relations between GER features, gastric emptying data and symptoms observed during the trial were statistically evaluated.

Results: During 90 hours of combined recording 248 reflux events (15.3\% acid) were detected in 30 neonates. 99 episodes were associated to symptoms. The average (SD) gastric emptying time was $148.2(21.9)$ minutes with a $\mathrm{T}^{1 / 2}$ of $56.2(15.1)$ minutes. The main gastric emptying velocity was $0.69(0.12)$ radians. The number of GER and acid GER per tracing were respectively $8.3(1.0)$ and $1.3(0.8)$. Crying(24) was associated to long and acid reflux events. Regurgitations(13) and vomiting(8) were associated to high level of GER and gastric filling level. Rumination(31) was increased during were associated to high level of GER and gastric filling level. Rumination(31) was increased during
high level GERs. Irritability(20) was observed in both: low level and high duration reflux episodes. In the end a correlation between apnea(3) and acid refluxes was observed.

Conclusions: Our data suggest a relationship between GER symptoms and gastroesophageal activity. These findings could be relevant to practice of giving neonatal GERD therapeutic treatments on the grounds of infants symptoms. Further studies are necessary to establish the impact of our results and a possible clinical utilization. 